Meta

Journal des tradlucteurs

Translators' Journal

Étiemble, le Jargon des sciences, Paris, Hermann, 1966, 184 p. [12 F]

\title{
Robert Dubuc
}

Volume 12, numéro 3, septembre 1967

URI : https://id.erudit.org/iderudit/003104ar

DOI : https://doi.org/10.7202/003104ar

Aller au sommaire du numéro

\section{Éditeur(s)}

Les Presses de l'Université de Montréal

\section{ISSN}

0026-0452 (imprimé)

1492-1421 (numérique)

Découvrir la revue

Citer ce compte rendu

Dubuc, R. (1967). Compte rendu de [Étiemble, le Jargon des sciences, Paris,

Hermann, 1966, 184 p. [12 F]]. Meta, 12(3), 94-96.

https://doi.org/10.7202/003104ar

Ce document est protégé par la loi sur le droit d'auteur. L'utilisation des services d'Érudit (y compris la reproduction) est assujettie à sa politique d'utilisation que vous pouvez consulter en ligne.

https://apropos.erudit.org/fr/usagers/politique-dutilisation/
Cet article est diffusé et préservé par Érudit.

Érudit est un consortium interuniversitaire sans but lucratif composé de l’Université de Montréal, l'Université Laval et l'Université du Québec à Montréal. Il a pour mission la promotion et la valorisation de la recherche. https://www.erudit.org/fr/ 


\section{LES \\ OUTILS \\ DU \\ TRADUCTEUR}

ÉTiemble, le Jargon des sciences, Paris, Hermann, 1966, 184 p. [12 F]

M. Étiemble, professeur à la Sorbonne, s'était livré, il y a quelques années ${ }^{1}$, à une charge à fond de train contre l'anglomanie des Français. Le voici qui revient à l'attaque en concentrant cette fois son tir sur le langage des sciences. Il dénonce dans ce nouvél ouvrage le jargon des hommes de science contemporains dont le langage tend de plus en plus à se couper de la langue commune. Il le fait dans un style qui tient plutôt du pamphlet que de l'analyse.

Ce livre n'a done pas un caractère scientifique. C'est l'œuvre d'un esthète $d u$ langage et non d'un linguiste de stricte observance. L'ouvrage se situe dans la respectable tradition de ces nombreux écrits qui, de Rivarol à Jacques Duron, se portent à la défense d'une langue vénérée à qui on veut conserver ses qualités essentielles.

Les abondants exemples que M. Étiemble nous met sous les yeux suffisent à nous faire partager son souei, sinon sa hargne, à l'égard de ce qu'on pourrait considérer comme les «déviations » du langage scientifique. L'honnête homme n'y trouve eertainement plus son compte.

\section{Le vocabulaire scientifique}

L'auteur dégage deux composantes fondamentales du langage des sciences: les néologismes et les glissements de sens. Il reconnaît aux savants le droit de se forger les outils verbaux dont ils ont besoin mais en prenant à son compte le principe énoncé par Le Lionnais: «L’idéal, pour le langage scientifique, serait de se tenir aussi près que possible du langage de la bonne vulgarisation, c'est-àdire du français commun. 》

1. Etiemble, Parlez-vous franglais ?, Paris, Gallimard, «Idées », 1964, 376 p. 
Voilà, semble-t-il, un premier principe à retenir pour l'orientation de toute terminologie technique si l'on veut que le langage reste un instrument de communication.

Malheureusement la tendance aujourd'hui semble tout autre. Le malaise tient pour une part au mépris qu'on affecte dans certains milieux scientifiques pour une forme honnête d'écriture. À ce propos, l'auteur cite une déclaration effarante d'un homme de science siégeant au jury d'une thèse de sciences naturelles : «Votre thèse, disait le savant au candidat, est trop bien écrite, et tout ce qui est bien écrit a toutes les chances de n'être qu'un tissu de mensonges. » Une telle affirmation met en bien mauvaise posture Ambroise Paré, Pascal, Descartes, Buffon, Lavoisier et Henri Poincaré! «Quelle aberration, dit Étiemble, de juger incompatibles une expression adéquate et la vérité scientifique!» Comment ne pas souscrire à cette défense du bon sens? L'auteur poursuit: «Plutôt penserais-je, pour l'avoir cent fois vérifié, que la clarté, l'élégance, sont aussi utiles dans les sciences qu'ailleurs et que tout texte médiocrement ou mal écrit révèle une imperfection de la pensée. »

\section{Snobisme}

Ce mépris de la forme correcte s'accompagne le plus souvent du culte de l'expression ésotérique inspirée soit de la «grécomanie » soit de l'« américanolâtrie». L'auteur n'est tendre ni pour l'une ni pour l'autre de ces formes d'《aberration». Il ne voit pas pourquoi on dirait « céphalalgie» au lieu de mal de tête, $\mathrm{ni}$ 《restless legs» pour impatiences, ni « environnement 》 pour milieu, ni « home care» pour soins à domicile.

Si les raisons qu'invoque l'auteur pour dénoncer ces abus n'ont pas toutes égale valeur - son antiaméricanisme ne semble pas tout à fait exempt de parti pris - il reste qu'une langue s'appauvrit en se laissant trop infiltrer par l'emprunt, pourvu qu'on admette qu'il puisse y avoir progrès ou recul dans l'évolution d'une langue. En laissant libre jeu à l'emprunt inutile, elle compromet l'équilibre de sa structure phonétique et affaiblit ses propres ressources terminologiques. Une langue grugée par l'emprunt risque de se trouver un jour en mauvaise posture. (Nous en savons quelque chose au Canada, et nons payons cher pour le savoir.)

\section{Motivation erronée des emprunts}

Le fait qu'une notion soit connue d'abord avec une étiquette étrangère établit entre cette désignation et. la chose une adéquation artificielle dont on ne s'affranchit pas facilement. Aucun vocable indigène ne semble avoir la précision de l'emprunt. Pourtant, à l'analyse, le terme emprunté n'a souvent dans sa langue d'origine aucune précision particulière. Tel est le cas du mót pattern qui est en anglais le strict équivalent du mot français patron. Les sens qu'on lui confère en français sont tout autant en puissance dans patron. On fait faire volontiers à l'emprunt une évolution sémantique qu'on refuse à son homologue de la langue emprunteuse.

De là à incriminer la pauvreté de cette dernière pour justifier l'emprunt il n'y a qu'un pas qu'on franchit allègrement. Toute langue a des lacunes que des emprunts judicieux peuvent combler. Mais la pauvreté prétendue du français 
tient souvent à l'ignorance des ressources de cette langue. C'est cette ignorance aussi qui fait qu'on accuse souvent à tort le français de manquer de concision. L'auteur donne une liste d'une quinzaine d'expressions techniques toutes plus concises en français qu'en anglais. Cela ne prouve pas que le français soit plus concis que l'anglais, mais que souvent la prolixité du français est attribuable à l'ignorance du terme exact.

\section{Néologismes}

Le problème de l'emprunt nous conduit à eelui des néologismes. L'évolution accélérée des techniques erée un besoin extraordinaire de mots nouveaux. Où prendre les matériaux pour désigner ces réalités nouvelles? À ce problème, l'auteur propose deux solutions: la première, c'est le recours aux parlers locaux, aux patois, au langage populaire, à l'argot des métiers; la seconde, c'est de constituer sur les familles morphologiques existantes des séries analogiques par la simple modification de la consonne initiale. Ainsi sur le modèle de l'adjectif creux et ses dérivés : creuser, creusage, creusement, creuset, creuseur, creusiste, creusoir, creusure, on pourrait former des familles analogiques avec breux, dreux, freux, greux et treux. En donnant au radical un sens précis et en respectant le sens du suffixe, on aurait là une source inépuisable de néologismes dont l'intégration à la langue ne poserait aucune difficulté formelle. Cependant la langue offre bien peu d'exemples de ces créations arbitraires qui ne soient pas mort-nées.

M: Étiemble nous laisse en conclusion certaines consignes à suivre à l'égard des emprunts: $" 1^{\circ}$ Traduire, inflexiblement, tous les mots anglais ou yanquis; $2^{\circ}$ Assimiler orthographiquement les mots étrangers dont nous ne savons plus nous passer. 》 Écrire par exemple jipe pour jeep, dachepot pour dash-pot.

Si l'ostracisme absolu de tous les vocables anglo-saxons semble une exagération de prosélyte, l'assimilation orthographique paraît à tout point de vue souhaitable en dépit des résistances qu'elle suscite.

RoBert Dubuc

\section{Documentation}

La Bibliothèque centrale de l'Université de Montréal a fait l'acquisition, sous forme de microfilms, d'une collection complète du Journal officiel de la République française. Les différentes parties de cette publication comprennent les 《Lois et décrets », les « Débats et documents parlementaires du Sénat », ainsi que les «Débats et documents de l'Union française ». Cette précieuse documentation, que d'aucuns considèrent unique au Canada, couvre les années 1869 à 1959. Des démarches sont en cours en vue d'une mise à jour, et déjà l'édition des «Lois et décrets »a été complétée jusqu'à 1965. On pourra d'ici peu consulter ces documents, au moyen d'une liseuse de microfilms, dans la salle M 605 récemment ouverte au public (Service des documents officiels).

(Communiqué) 